\title{
Is Cholecystectomy Necessary After ERCP for Bile Duct Stones in Patients with Gallbladder in situ?
}

Soon Kil Kwon, M.D., Byung Seok Lee, M.D.*, Nam Jae Kim, M.D.*, Heon Young Lee, M.D.*, Hee Bok Chae, M.D., Sei J in Youn, M.D. and Seon Mee Park, M.D.

Departments of Internal Medicine, Chungbuk National University College of Medicine and Medical Research Institute, Cheongju, Ko rea;

Departments of Internal Medicine*, Chungnam National University College of Medicine, Daejeon, Ko rea

Backg round : The requirement for subsequent cholecystectomy in patients $w$ ith gallbladder in situ after endoscopic removal of stones from the com mon bile duct $(C B D)$ is controversial. The aims of this study were to assess the requirement for subsequent cholecystectomy for gallbladder-related symptoms, and to identify the patients who develop symptoms after the endoscopic removal of CBD stones.

Methods : Of 241 patients $w$ ith gallbladder in situ following endoscopic removal of stones from the CBD, 146 patients $(78$ men and 68 women; mean age $69 \pm 13$ years, range 20-93) with a follow-up time of more than three months without elective cholecystectomy were enrolled in the study. Fifty-nine patients had gallbladder stones (single stones in 27 and multiple stones in 32) and 87 patients had gallbladder in situ $w$ ithout stones. The time from entry to the occurrences of death or cholecystectomy was evaluated retrospectively. Cox regression analys is $w$ as used to evaluate the ris $k$ factors associated $w$ ith these events.

Res ults : The mean duration of follow-up was $24.1 \pm 18.0$ months (range $3-70$ months). During follow-up, seven patients (4.8\%) underwent cholecystectomy, on average 18.4 months after CBD stone removal, as the result of acute cholecystitis in four cases, biliary pain in two cases and acute pancreatitis in one case. Laparoscopic cholecystectomy was performed in four patients and open cholecystectomy in three patients. Post-operative morbidity occurred in two patients, with improvement after conservative management. Nine patients (6.2\%) died as the result of un related biliary disease. Age, sex, presence of gallbladder stones, multiplicity of gallbladder stones and underlying disease did not correlate with subsequent cholecystectomy by Cox regression analys is.

Conclus io $\mathbf{n}:$ Elective cholecystectomy is not warranted in patients $w$ ith bile duct stones when the common duct can be cleared of stones by endoscopic sphincte rotomy. We could not find any clinical predictors of further sy $m$ to $m s$ or complications arising from the retained gallbladder.

Key Words : Gallbladder in situ, cholecystectomy, common bile duct calculi, Sphincterotomy, endoscopic.

\section{INT RO DUCT IO N}

Endoscopic sphincterotomy (EST) is a safe and effective treatment for bile duct calculi, ${ }^{1,2)}$. At present, EST is

Address reprint requests to : Seon Mee Park, M.D.,

Department of Intemal Medicine, Chungbuk National

University College of Medicine, San 48, Gaeshin-dong,

Hungduk-ku, Cheongju, Korea, 361-763. advocated in most patients with bile duct stones and in patients with gallbladder (GB) in situ, regardless of age $e^{3,4)}$ The requirement for cholecystectomy after EST in patients with common bile duct (CBD) stones and GB in situ is still the subject of controversy. The incidence of symptomatic cholecystectomy for the conditions of acute cholecystitis, acute pancreatitis and abdominal pain after EST varies from $2.5 \%$ to $22 \%^{5,6)}$. The predictive factors for the 
development of biliary symptoms after EST in patients with intact gallbladders have not yet been defined. Some authors recommend elective cholecystectomy after EST in cases of GB calculi, preexisting cholangitis, acute biliary pancreatitis, complete opacification of the GB during endoscopic retrograde cholangiopancreatography (ERCP) and nonvisualization of the GB after EST, but others do $\operatorname{not}^{7,8,9,10)}$. Therefore, clinical outcomes at follow-up and identification of factors' subsequent symptoms from the biliary tract are needed.

In this study, we observed the clinical progress of patients after endoscopic removal of CBD stones, until subsequent cholecystectomy or death, to evaluate the need for cholecystectomy in patients with gallbladder in situ after endoscopic treatment for bile duct stones. We also analyzed the characteristics of the patients who required cholecystectomy to ascertain the predictive factors for subsequent cholecystectomy.

\section{MATERIALS AND METHODS}

\section{Patients}

Patients enrolled in this study met the following criteria: (1) ERCP with EST performed between March 1995 and December 2000 in the Chungbuk National University Hospital or Chungnam National University Hospital (2) the presence of CBD stones and the ir complete clearance documented by cholangiography (3) older than 20 years of age at the initial ERCP (4) GB in situ (with or without stones) at the initial ERCP documented by abdominal ultrasonography (5) no evidence of underlying malignancy or intrahepatic duct (IHD) stones, and (6) follow- up time of more than three months after ERCP.

\section{Methods}

This study was conducted retrospectively using chart reviews and interviews by telephone. The protocols were approved by the Chungbuk National University Ethical Committee and the Chungnam National University Ethical Committee. The entry date was the time of complete CBD stone removal by ERCP and EST. The follow- up times were determined from the entry date to the occurrence of subsequent cholecystectomy or death. During follow-up, the occurrence of symptoms or signs related to biliary tract stones (such as biliary pain, gallstone pancreatitis, cholangitis, recurrences of biliary stones and biliary tract cancers) and major operations unrelated to biliary tract disease were recorded. Serum bilirubin, transaminase, alkaline phosphatase and the presence of GB stones or sludge as detected by abdominal ultrasound were recorded at the time of entry. The causes of death for those patients who died during the study were analyzed, and whether they were related to biliary tract disease or not was determined. For patients who required subsequent cholecystectomy, we evaluated the causes of the cholecystectomy and the characteristics of hospitalization, such as the operation method (laparoscopic cholecystectomy or open cholecystectomy), morbidity and mortality. Age, sex, underlying disease, the presence of gallbladder stones and the multiplicity of gallbladder stones were analyzed as possible risk factors in the cases of subsequent cholecystectomy.

\section{Statistical analysis}

Results are expressed as means SD. Statistical analyses were performed using a one-way ANOVA test for continuous variables and a chisquared test for categorical variables. The risk factors for developing biliary tract-related symptoms requiring subsequent cholecystectomy were analyzed by Cox regression. A value of $p<0.05$ was considered statistically significant.

\section{RES ULTS}

During the study period, 363 patients with CBD stones underwent ERCP and EST. Of these, 141 patients $(40.6 \%)$ were excluded from the study because of failure of stone removal in four patients, cholecystectomies before ERCP in 63 patients, concomitant IHD stones in 26 patients, incomplete medical records for six patients, underlying malignancies in six patients, GB polyps in one patient and loss of follow-up or follow-up of less than three months in 35 patients.

Of 222 patients with GB in situ, 76 underwent elective cholecystectomy after ERCP (elective cholecystectomy group) and the 146 patients who did not undergo cholecystectomy were enrolled in this study ("wait-andsee" group) (Table 1). The "wait-and-see" group was divided into 59 patients with GB stones (single stones, $\mathrm{n}=27$; and multiple stones, $\mathrm{n}=31$ ) and 87 patients without stones. Of the 76 patients of the elective cholecystectomy group, 68 had GB stones (single stones, $\mathrm{n}=25$; and multiple stones, $\mathrm{n}=43$ ) and eight had GB sludge. The re were no significant differences among the three groups in sex, the percentage of associated 
disease, liver function tests at the initial ERCP or indications for the initial ERCP. The patients of the elective cholecystectomy group were younger than those of the "wait-and-see" group $p<0.001$ ). At the time of entry, 80 patients complained only of abdominal pain, 119 patients of cholangitis, 11 patients of acute cholangitis and 15 patients of nonspecific upper gastrointestinal symptoms. The mean values of physiological parameters were: peripheral blood leukocytes, $9619 / \mathrm{mm}^{3}$; total serum bilirubin concentration, $3.9 \mathrm{~g} / \mathrm{dL}$; serum alanine transferase level, $186 \mathrm{U} / \mathrm{L}$; and serum alkaline phosphatase level, $509 \mathrm{U} / \mathrm{L}$ These values returned to normal within three weeks after CBD stone removal.

Twenty-two elective cholecystectomy patients (28.9\%) underwent open cholecystectomy and 54 (71.1\%) underwent laparoscopic cholecystectomy within one month of the clearance of bile duct stones. The follow- up time in the "wait-and-see" group, from entry to the time of death, cholecystectomy or the end of the study, was $24.2 \pm 17.7$ months (range 3-70 months). During follow- up, 12 patients (8.2\%) (seven men and five women; mean age $69 \pm 15$ years, range 35-93 years) required repeated ERCP for the recurrence of bile duct stones and managed ERCP alone. One patient was admitted for recurrent acute pancreatitis and underwent cholecystectomy (Table 3, patient 7). Biliary-related symptoms developed in 13 patients. Five patients (3.4\%) suffered from mild upper abdominal pain but did not undergo cholecystectomy, and seven patients (4.8\%) underwent cholecystectomy (Table 2). Subsequent cholecystectomies were performed

Table 1. Characteristics of the patients enrolled in this study

\begin{tabular}{|c|c|c|c|c|}
\hline & \multirow{2}{*}{$\begin{array}{c}\text { Elective } \\
\text { Cholecystectomy } \\
(\mathrm{n}=76)\end{array}$} & \multicolumn{2}{|c|}{ "Wait- and-See" } & \multirow[b]{2}{*}{$p$ value } \\
\hline & & $\begin{array}{c}\text { Stone }(+) \\
(\mathrm{n}=59)\end{array}$ & $\begin{array}{c}\text { Stone }(-) \\
(n=87)\end{array}$ & \\
\hline \multicolumn{5}{|l|}{ GB stone } \\
\hline (s ingle/multiple/s ludge) & $25 / 43 / 8$ & $27 / 31 / 0$ & $0 / 0 / 0$ & $<0.001$ \\
\hline Age (year) & $58 \pm 16(20-83)$ & $70 \pm 14(20-93)$ & $69 \pm 12(35-93)$ & $<0.001$ \\
\hline $\operatorname{Sex}(M: F)$ & $44: 32$ & $32: 27$ & $46: 41$ & NS \\
\hline \multicolumn{5}{|l|}{ Indication for ERCP } \\
\hline Abdominal pain & 35 & 16 & 29 & \\
\hline Cholangitis & 37 & 37 & 45 & \\
\hline Acute pancreatitis & 3 & 2 & 3 & \\
\hline Others & 1 & 4 & 10 & \\
\hline \multicolumn{5}{|l|}{ Initial ERCP } \\
\hline WBC & $9858 \pm 4801 / \mathrm{mm}^{3}$ & $8559 \pm 4544 / \mathrm{mm}^{3}$ & $10133 \pm 4794 / \mathrm{mm}^{3}$ & NS \\
\hline Bilirubin (total) & $3.5 \pm 2.5 \mathrm{mg} / \mathrm{dL}$ & $4.8 \pm 4.7 \mathrm{mg} / \mathrm{dL}$ & $3.5 \pm 2.6 \mathrm{mg} / \mathrm{dL}$ & NS \\
\hline Ala nine transferase & $221 \pm 242 \mathrm{U} / \mathrm{L}$ & $163 \pm 149 \mathrm{U} / \mathrm{L}$ & $169 \pm 174 \mathrm{U} / \mathrm{L}$ & NS \\
\hline Alka line phosphatase & $534 \pm 420 \mathrm{U} / \mathrm{L}$ & $498 \pm 503 \mathrm{U} / \mathrm{L}$ & $495 \pm 273 \mathrm{U} / \mathrm{L}$ & NS \\
\hline
\end{tabular}

Table 2. Seven patients who need subsequent cholecystectomy

\begin{tabular}{cccccccc}
\hline No. & Age/Sex & Operation & GB stone & $\begin{array}{c}\text { Duration } \\
\text { (month) }\end{array}$ & Associated & Causes & Pathology \\
\hline 1 & $76 / \mathrm{F}$ & Open & Single & 6 & DM & Acute cholecystitis & Acute \& chronic cholecystitis \\
2 & $74 / \mathrm{F}$ & LC & Multiple & 17 & None & Abdominal pain & Chronic cholecystitis \\
3 & $60 / \mathrm{M}$ & Open & Multiple & 24 & Af & Acute cholecystitis & Acute \& chronic cholecystitis \\
4 & $76 / \mathrm{M}$ & LC & Single & 30 & None & Abdominal pain & Chronic cholecystitis \\
5 & $71 / \mathrm{M}$ & Open & No stone & 11 & CGN & Acute cholecystitis & Acute gangrenous cholecystitis \\
6 & $69 / \mathrm{M}$ & LC & No stone & 23 & DM & Acute pancreatitis & Gangrenous cholecystitis \\
7 & $68 / \mathrm{M}$ & LC & No stone & 40 & None & Acute cholecystitis & Acute gangrenous cholecystitis \\
\hline
\end{tabular}

LC, Laparoscopic cholecystectomy 
Table 3. Nine patients died during follow up

\begin{tabular}{cclccc}
\hline No. & Age/Sex & GB stone & Duration (month) & Associated disease & Causes of Death \\
\hline 1 & $80 / \mathrm{M}$ & Multiple & 2 & None & Old age \\
2 & $83 / \mathrm{M}$ & Single & 3 & None & Old age \\
3 & $75 / \mathrm{M}$ & Single & 9 & Dementia & Old age \\
4 & $78 / \mathrm{M}$ & Multiple & 15 & None & Old age \\
5 & $86 / \mathrm{F}$ & Multiple & 15 & HTN, DM & Old age \\
6 & $82 / \mathrm{M}$ & No stone & 3 & None & Pnemuonkia \\
7 & $63 / \mathrm{M}$ & No stone & 10 & Alcoholic LC (C) & Variceal bleeding \\
8 & $85 / \mathrm{M}$ & No stone & 22 & Tbc & Traffic accident \\
9 & $76 / \mathrm{F}$ & No stone & 40 & None & Cellulitis \\
\hline
\end{tabular}

on average 18.4 months (range 140 months) after the removal of CBD stones (Figure 1) because of acute cholecystitis $(n=4)$, biliary pain $(n=2)$ or acute pancreatitis $(n=1)$. Of the seven cholecystectomies, three $(42.9 \%)$ were open procedures. There was no mortality related to biliary disease. Nine patients $(6.2 \%)$ died of causes unrelated to biliary disease 240 months after entry (Table 3).

\section{DIS C US S IO N}

Before the introduction of EST, bile duct stones and gallbladder in situ were treated in a single procedurebile duct exploration and cholecystectomy ${ }^{8)}$. At present, patients are treated first with EST and cholecystectomy is performed later in patients with retained GB stones ${ }^{11}$. Even in the absence of GB stones, some practitioners recommend cholecystectomy for conditions including complete GB opacification or non-visualization during ERCP, acute biliary pancreatitis and pre-existing cholangitis $^{12)}$. This study was designed to determine the treatment strategy for GB in situ after clearance of bile duct stones by ERCP. The expected disadvantages of the wait-and-see strategy were the development of GB-related symptoms and post-operative complications related to the symptomatic cholecystectomy. The present study demonstrates that the incidence of subsequent cholecystectomies is low and operative mortality and morbidity are very low, indicating that prophylactic cholecystectomy is not required in patients with cleared bile duct stones and GB in situ. These results are consistent with those of various studies that report subsequent cholecystectomy in 5.2-22.0\% of patients ${ }^{6,9)}$. As most symptoms disappear after ERCP, cholecyst- ectomy was intended to prevent the symptoms associated with GB in situ. After EST, GB contractility is enhanced and bile lithogenicity decreased by prolonged nucleation time and reduced cholesterol saturation index ${ }^{13)}$, so newly developed GB calculi are minimal. Furthermore, GB stones that have never caused symptoms are unlikely to cause symptoms in the future ${ }^{6,14)}$. Stone composition and pathogenesis differ between GB calculi and bile duct stone ${ }^{13)}$, so in cases of cleared bile duct stones and resolved symptoms, GB calculi are asymptomatic ${ }^{15,16)}$. The remaining GB stones cause symptoms at a later date in only a small percentage of patients. There was no higher risk of complications in the remaining gallbladder than in cases without $\mathrm{EST}^{17)}$.

Risk factors reported for subsequent cholecystectomy include the presence of GB stones, cystic duct obstruction and complete opacification of GB during ERCP. Of these, the most frequently reported risk factor is the presence of GB stones ${ }^{6,7,12)}$. Therefore, we investigated whether the presence of GB calculi has an effect on the development of GB-related symptoms and symptomatic cholecystectomy. Our results show that the rates of subsequent cholecystectomy are not different for patients with or without GB calculi. CBD stones develop in old age, and the morbidity associated with cholecystectomy may be greater than in normal cholecystectomy. The requirement for cholecystectomy in this situation must be considered. In young patients, cholecystectomy is usually recommended for the prevention of biliary-related symptoms in the future ${ }^{3)}$. In our study, however, 70 patients $(47.9 \%)$ less than 70 years of age belonged to the "wait-and-see" group. Of these, only three underwent subsequent cholecystectomy, and they improved without post-operative morbidity or mortality. Therefore, we 
consider that the "wait-and-see" strategy can be extended to young patients. In the absence of GB stones, recurrent biliary symptoms may originate from acalculus cholecystitis . Therefore, we evaluated the relationship between the development of symptoms and underlying disorders, such as diabetes mellitus, heart diseases and cerebrovascular accidents. The presence of underlying diseases had no influence on the development of biliary symptoms.

How are we to understand the cases of combined cholangitis and cholecystitis at the initial ERCP? We do not precisely differentiate the patients with combined cholecystitis and cholangitis. However, complete improvement in the clinical symptoms and laboratory data after bile duct stone removal suggests that the main symptoms originated from the bile duct stones and most GB calculi were asymptomatic. After an initial attack of acute biliary pancreatitis, early cholecystectomy is usually recommended to prevent further episodes ${ }^{7)}$. Recently, many studies have shown that the chance of recurrent pancreatitis is low, and that attacks are often mild ${ }^{14)}$. In this study, only three patients underwent EST for acute biliary pancreatitis, and one patient required re-ERCP and cholecystectomy. Because the sample size is too small, it is difficult to conclude from these data.

Our study has limitations due to the short time of follow- up, with $30 \%$ less than 12 months. Furthermore, this study was conducted retrospectively, so cystic duct obstruction and the origin of bile duct stones (whether they migrated from the GB or were synthesized de novo) are not fully evaluated. In cases in which CBD stones migrate from the GB, the possibility of developing biliary symptoms may be high. However, the proportion of stones migrating from the GB is usually $10-15 \%$ and it is difficult to determine the origin of bile duct stones in a clinical setting.

The patients enrolled in this study improved after the endoscopic removal of bile duct stones and later cholecystectomy for biliary symptoms was required in only a small number of patients and did not entail serious complications. We, therefore, recommend that cholecystectomy be restricted to patients with symptoms from GB stones and that the indefinite postponement of cholecystectomy may be warranted in patients with GB in situ, for whom endoscopic treatment may be sufficient.

\section{REFERENCES}

1. Materia A, Pizzuto G, Silecchia G, Fiocca F, Fantini A, Spaziani E, Basso N. Sequential Endoscopic-Laparoscopic
Treatment of Cholecystocholedocholihiasis. Surg. Laparos copy and Endoscopy 6(4)273-277, 1996

2. Bergamaschi R, Tuech JJ, Braconier L, Walsoe HK, Marvik R, Boyet J, Arnaud JP. Selective Endoscopic Retrograde Cholangiography prior to Laparoscopic Cholecystectomy for Gallstones. The Am. J. of Surg. 178(1): 46-49 Jub 1999

3. Jacques JGHM Bergman, Suzanne van der Mey, Eric AJ Rauws, Jan GP Tijssen, Dirk-Jan Gouma, Guido N Tytgat, Kees Huibregtse. Long-term follow-up after endoscopic sphincterotomy for bile duct stones in patients younger than 60 years of age. Gastrointestinal Endoscopy 44, No. 6: 643-649 1996

4. Sugiyama M, Atomi Y. Follow- up of more than 10 years after endoscopic sphincterotomy for chole lithiasis in young patients. The British J. of Surg. 85(7): $917-9211998$

5. Tanaka M, kkeda S, Yoshimoto $\mathrm{H}$, Matumoto S. The long-tem fate of the gallbladder after endoscopic sphincterotomy. Complete follow- up study of 122 patients. Am. J. Surg. 154(5): 505-509 Nov 1987

6. Eric Kullman, Kurt Borch, Lars-Gran Dahlin and gustav Liedberg. Long-Term Follow-Up of Patients with Gallbladder in situ After Endoscopic Sphincterotomy for Choledocholithiasis. Eur. J. Surg. 157:13 1- 135, 1991

7. LE Hammarstrm, T Holmin \& H Stridbeck. Endoscopic Treatment of Bile Duct Calculi in Patients with Gallbladder in Situ - Long-Tem Outcome and Factors Predictive of Recurrent Systems. Scand. J. Gastroenterol. 31294-301, 1996

8. Ingoldby CJ, El-Saadi J, Hall RI, Denyer ME. Late Results of Endoscopic Sphincterotomy for Bile Duct Stones in Eldeny Patients with Gall Bladder in situ. Gut 30(8) : 129-1131, Aug 1989

9. J. Hill DF, Martin and DEF Tweedle. Risks of Leaving the Gallbladder in situ after Endoscopic Sphincterotomy for Bile Duct Stones. Br. J. Surg. Vol. 78:554-557, May 1991

10. Harry S. Himal, MD, FRCSC, FACS. Role of Endoscopic Sphincterotomy Alone in Patients with Cholesocholithiasis and Cholelithiasis. CJS, Vol. 39, No. 3225-228, June 1996

11. D. T. Hansell, M. A. Millar, W. R. Murray, G. R. Gray and G. Gillespie. Endoscopic Sphincterotomy for Bile Duct Stones in Patients with Intact Galbladders. Br. J. Surg. Vol. 76:856-858, August 1989

12. Sharma BC, Agarwal DK, Ba jal SS, Negi TS, Choudhuri G, Saraswat VA. Effect of Endoscopic Sphincterotomy on Gall Bladder Bile Lithogenicity and Motility. Gut 42(2):288-292, Feb 1998

13. Yolande CA Keulemans, MD, PhD, Erik AJ Rauws, MD, $\mathrm{PhD}$, Kees Huibregtse, MD, PhD, Dirk J Gouma, MD, $\mathrm{PhD}$. Current Management of the Gallbladder after Endoscopic Sphincterotomy for Common Bile Duct Stones. Gastrointest. Endosc. 46: 5 4-5 19, 1997 
14. FX Caroli- Bosc, JC Montet, L Salmon, JF Demarquay, R Dumas, AM Montet, JL Bernard, JP Delmont. Effect of Endoscopic Sphincterotomy on Bile Lithogenicity in Patients with Gallbladder in situ. Endoscopy 3 1(6): 437-44 1, 1999

15. EM Targa rona, I Pros M Trias. Effects of Sphincterotomy on Gallbladder Physiology: A Review. Endoscopy 27: 388-391, 1995
16. Kwok- Hung Lai, MD, Liang-Feng Lin, MD, Gin- Ho Lo, $\mathrm{MD}$, J in-Shiung Cheng, MD, Rong-Long Huang, MD, Chiun- Ku Lin, MD, Jia-Sheng Huang, MD, Ping- I Hsu, MD, Nan-Jing Peng, MD, Lu-Ping Ger, MPH. Does Cholecystectomy after Endoscopic Sphincterotomy Prevent the Recurrence of Biliary Complications? Gastrointest. Endosc. 49:483-487, 1999 